\title{
NASAL ENDOSCOPE FOR TYPE 1 TYMPANOPLASTY; PROS \& CONS - AN ANALYTICAL STUDY
}

\author{
Shibu George ${ }^{1}$ \\ ${ }^{1}$ Additional Professor, Department of ENT, Government Medical College, Trivandrum.
}

\begin{abstract}
\section{BACKGROUND}

Minimally invasive ear surgery has developed along with endoscopic techniques; this avoids other unnecessary incisions and soft tissue dissections. The endoscopic approach also provides better visualisation of hidden areas in the middle ear cavity and provides better visualisation of structures parallel to the axis and beyond the shaft of the instruments that otherwise can cramp surgery using a microscope. One major disadvantage of using a smaller-diameter endoscope in the ear is a narrow and tubular viewing. The use of a traditional $4 \mathrm{~mm}, 18 \mathrm{~cm}$ nasal endoscope could effectively negate this major disadvantage by providing a close-up wide-angled vision.
\end{abstract}

\section{MATERIALS AND METHODS}

This was a prospective 4-year study of endoscopic type 1 tympanoplasty, using 4-mm $0^{0}$ nasal endoscope, in 35 patients with chronic otitis media (COM), having inactive mucosal disease. Per-operative parameters and post-operative results were analysed by standard proforma.

\section{RESULTS}

There was satisfactory graft uptake in $83.86 \%(n=29)$ cases with good air-bone gap closure. $17.14 \%(n=6)$ of patients complained of thermal irritation in the ear during surgery. Post-operative infection rate was on the higher side (22.86\%). Operating time was comparable to microscopic tympanoplasty.

\section{CONCLUSION}

The nasal endoscope provides unmatched middle ear visualisation and image quality with significant benefits in teaching with comparable results to microscopic tympanoplasty. Light source should be used in lower setting to prevent thermal irritation to the ear. Meticulous sterilisation of the endoscope is prudent to prevent post-operative infection.

\section{KEYWORDS}

Tympanoplasty, Nasal Endoscope, Chronic Otitis Media, Middle Ear.

HOW TO CITE THIS ARTICLE: George S. Nasal endoscope for type 1 tympanoplasty; pros \& cons - An analytical study. J. Evolution Med. Dent. Sci. 2017;6(3):146-149, DOI: 10.14260/Jemds/2017/36

\section{BACKGROUND}

Although it has been a long time since the introduction of operative endoscopy in surgeries of the middle ear, ${ }^{[1,2]}$ still limited space has been advocated for the endoscopic management of middle ear disease earlier. Starting in the 1990s, this gained more popularity in otologic surgery, and significantly changed not only surgical concepts but also anatomic and physiologic concepts, and has become increasingly popular during the last 15 years. $[3,4,5]$ The operating microscope had enjoyed cult status in almost all middle ear surgeries to date and it provides a very good quality magnified image in a straight line; however, the surgeon's field of view is limited and sometimes cumbersome to operate using trans-canal approach, especially with added anatomical constraints unless the surrounding bone is removed for suitable access. Such sites may include the anterior recess of external canal, medial attic, supratubal recess, posterior mesotympanum (including sinus tympani,

Financial or Other, Competing Interest: None.

Submission 19-12-2016, Peer Review 30-12-2016,

Acceptance 02-01-2017, Published 07-01-2017.

Corresponding Author:

Dr. Shibu George,

Charivaukalayil,

Ettumanoor P. $O$.

Kottayam-686631, Kerala.

E-mail: drshibugeorge@gmail.com

DOI: $10.14260 /$ jemds $/ 2017 / 36$ facial recess) and hypotympanum. As opposed to operating microscope, the endoscope doesn't require wide viewing portals for adequate illumination and visualisation, instead provides direct illumination and vision of the operating field which effectively overcomes the necessity for extra exposure by drilling. Furthermore, there is better visualisation of structures parallel to the axis and beyond the shaft of the instruments that otherwise can cramp microscopic surgery for space. Adequate magnification can be simply achieved here by just getting the endoscope closer to the surgical field. $[6,7,8,9,10]$

Ironically, the endoscope has not taken off as flamboyantly as it did in the nose and paranasal sinuses where it continues to rule the roost. There are several possible reasons for this such as the longer learning curve taken to adapt to newer instrumentation, loss of depth perception due to monocular/projected viewing and use of one hand for holding the endoscope.[10] Still the other advantages would have cancelled off all these hitches but for one major disadvantage of using a smaller-diameter endoscope in the ear, namely a narrow and tubular viewing. This effectively cancels out the advantage of wider field of view when compared with the microscope. The use of a traditional $4 \mathrm{~mm}, 0^{0}$ wide-angled nasal endoscopes could effectively negate this major disadvantage by providing a magnified vision that enables to change rapidly from a closeup to a wide-angle view, just by going closer or by withdrawing the instrument. Thus, an all-encompassing view 
of the 3 key areas of tympanoplasty surgery is possible even in the presence of an overhang; namely the canal, tympanic membrane and the annulus. Further, it provides an all-round vision to the surgeon who can rotate angled endoscopes to visualise the deep and hidden structures within the middle ear as well. $[9,10]$

This is a preliminary report and a critical analysis on the experience of the author with 35 cases of endoscopic tympanoplasty over 4 years, performed using wide-angle $0^{0}$ nasal endoscope in an attempt to realise the advantages, disadvantages and problems specific for the same.

\section{MATERIALS AND METHODS}

This prospective study was done in 35 patients, between December 2009 to November 2013 in the Department of ENT, Govt. Medical College, Kottayam after obtaining clearance from the institutional review board and informed patient consent.

\section{Inclusion Criteria}

Patients with chronic otitis media (COM), inactive mucosal disease with adequate cochlear reserve, aged between 12 years and 60 years were included in the study.

\section{Exclusion Criteria}

Patients with COM having active ear discharge, evidence of ossicular disruption, ossicular fixity, poor cochlear reserve, squamosal disease or complications were excluded. Patients with COM with very narrow external auditory canal and external ear pathologies like otitis externa or osteoma were also excluded.

Conditions predisposing to active ear infection like tonsillopharyngitis, rhinosinusitis and nasal allergy were treated appropriately before taking up for surgery. All patients underwent pre-operative hearing assessment by tuning fork testing and pure tone audiometry to assess the air-bone gap and cochlear reserve.

The surgery was performed under local anaesthesia in all cases. A $4 \mathrm{~mm}$ wide-angled $0^{0}$ Hopkins telescope for routine nasal surgeries was used in all the cases. A $2.7 \mathrm{~mm}$ ototelescope (With a narrower field of view) was kept as a supplement to enable use in presence of very narrow canals. All procedures were performed directly off the monitor positioned across the patient's head. A Mayo's stand supported the camera and endoscope. An absorbent pad soaked in antifog solution was kept above the head of the patient. The instruments used were all the same as those used in conjunction with microscopic ear surgery including micro ear knives, picks, curettes, elevators scissors and forceps. After adequate premedication, four quadrant infiltration of the external auditory canal was done with $2 \%$ Xylocaine $+1 / 100000$ adrenaline injection about $3 \mathrm{~mm}$ from the annulus. Temporalis fascia graft was harvested through a $2 \mathrm{~cm}$ incision above the helix within the hairline and made to dry. Margins of perforation was freshened using a sickle knife and under surface scraped with a curette to remove squamous epithelium. Adhesions visualised between the squamous margins of the tympanic membrane with middle ear mucosa were released and myringosclerotic patches excised. Tympanomeatal flap was elevated using a Rosen's knife up to the annulus by applying pressure to the bone. Annulus was elevated and gradually lifted from its rim after incising the middle ear mucosa. The flap was pushed anteriorly till the handle of the malleus became visible. It was freed from the handle of malleus by sharp dissection; medialised malleus handle if present was lateralised after releasing adhesions with promontory and if needed nipping off the tip. The handle of the malleus was thus fully stripped of its mucosal covering. The integrity and mobility of the ossicular chain was confirmed by direct inspection and palpation. The temporalis fascia graft cut to appropriate size was introduced under the tympanomeatal flap which had been elevated, insinuating it under the handle of malleus. The flap was repositioned so that that it covered the free edge of the graft which had been introduced. Bits of gel foam were placed around the edges of the raised flap and over the sealed perforation. In the absence of adequate eustachian tube function, gel foam was also kept in the middle ear to support the flap thus preventing medialisation of the graft.

All patients were called for regular post-operative follow-up; fortnightly for 6 weeks, every month for 6 months and every 2 months till 1 year. Hearing assessment with tuning fork tests and pure-tone audiometry was done by about 4-6 weeks (once the neo-tympanum was formed) and repeated after 3 months.

\section{RESULTS}

A total of 35 cases were included, of these 21 were male and 14 were female. The age range was from 17 to 51 years and mean age was 29.23 years. (Table 1 ).

The operating time needed for the procedure ranged between 55 minutes to 92 minutes with an average time of 74 minutes.

6 patients complained of intolerance due to thermal irritation generated from the endoscope during the procedure.

$82.86 \%$ of study population $(n=35)$ had full graft take-up and survival beyond 6 months while there was graft rejection in $17.14 \%$.

4 patients developed graft medialisation after complete take-up; all of them occurred after 6 months following surgery.

There was no difference in outcome between anterior and posterior quadrant perforations. Mean pre-operative air conduction threshold was $33.25 \pm 10.12 \mathrm{~dB}$ whereas Mean post-operative air conduction threshold was $22.92 \pm 6.07 \mathrm{~dB}$. Mean air bone gap pre-operatively was $23.63 \pm 5.98 \mathrm{~dB}$ and post-operatively was $12.34 \pm 6.27 \mathrm{~dB}$.

8 patients developed discharge from the ear after surgery; this occurred immediately after surgery in 5 and delayed by 2-3 months in the remaining 3 patients.

Transient giddiness was experienced by 3 patients in the immediate post-operative period which subsided with conservative management. I patient complained of tinnitus in the ear for 2 days which subsided spontaneously. (Table 2)

\begin{tabular}{|c|c|c|c|}
\hline Age Group & Males & Females & Total \\
\hline $11-20$ & 4 & 3 & 7 \\
\hline $21-30$ & 8 & 5 & 13 \\
\hline $31-40$ & 6 & 3 & 9 \\
\hline $41-50$ & 2 & 2 & 4 \\
\hline $51-60$ & 1 & 1 & 2 \\
\hline T0TAL & 21 & 14 & 35 \\
\hline \multicolumn{4}{|c|}{ Table 1. Age \& Sex Distribution of Study Population } \\
$(n=35)$
\end{tabular}




\begin{tabular}{|c|c|c|c|c|c|c|}
\hline 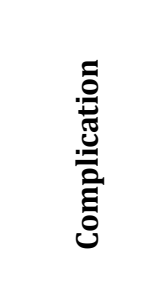 & 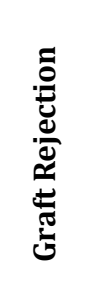 & & 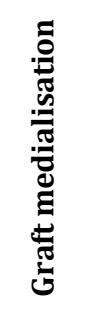 & 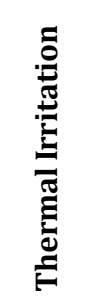 & 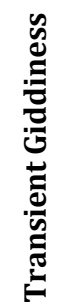 & $\stackrel{\mathscr{O}}{\stackrel{\Xi}{\Xi}}$ \\
\hline $\begin{array}{c}\text { No. of } \\
\text { Patients }\end{array}$ & 6 & 8 & 4 & 6 & 3 & 1 \\
\hline $\begin{array}{l}\text { Percentage } \\
\quad(\%)\end{array}$ & 17.14 & 22.86 & 11.43 & 17.14 & 8.57 & 2.86 \\
\hline \multicolumn{7}{|c|}{$\begin{array}{l}\text { Table 2. Complications Observed during/following } \\
\text { Endoscopic Tympanoplasty }\end{array}$} \\
\hline
\end{tabular}

\section{DISCUSSION}

The graft take-up rate was comparable to that of microscopic surgery; 29 out of 35 cases had full graft take-up and survival beyond 6 months. Of the 6 cases of graft rejection, 1 occurred in the immediate post-op period, 2 cases within 3 months and 3 cases after 3 months. 3 cases developed rejection along with discharge from the ear, while 2 cases presented as spontaneous perforation of the taken-up graft noted during routine follow-up. Post-operative infection rate was found to be on the higher side in endoscopic tympanoplasty using the nasal endoscope. 8 of the 35 patients developed discharge from the ear after surgery. Of the 8 patients, 5 developed discharge immediately after surgery during the $1^{\text {st }}$ week while remaining 3 had infection after initial graft take by 2-3 months. Culture was negative in all 3 of the 8 cases of delayed infection; from the immediate cases, mixed flora was isolated in 2, Staphylococcus aureus in 1 and was negative in remaining 2 . Clearly the immediate infection points to the possible risk of using the nasal endoscope which might have harboured nasal pathogens and hence warrants enhanced sterilisation techniques for the endoscope. Interestingly, 4 out of 5 cases of early discharge settled with antibiotics with proper graft take-up; on the contrary all 3 cases with late discharge developed graft rejection pointing more towards added host factors being responsible here than pure infection. The medialisation noticed in 4 patients probably reflect the poor eustachian tubal status of these patients. There was no significant difference in the operating time as compared to the microscope as it was case dependent. Postop morbidity possibly was less since transcanal route was employed in all patients avoiding unnecessary incisions.

The quality of the endoscopic image was excellent and so was the all-encompassing visualisation of the tympanic cavity through the perforation. As opposed to the microscope, there was no need to manipulate the head of the patient for adequate viewing. The middle ear could be easily examined using the endoscope with close-up and wide-angle view possible just by going closer or by withdrawing the instrument; effectively this addresses the adequacy of magnification $[9,10]$. This unravelled majority of the middle ear anatomical blind areas like the foot plate area, facial nerve, cochleariform process, tensor tympani tendon, sinus tympani, tympanic plexus, \& eustachian tube. Ossicular integrity, mobility and tympanosclerosis were much easier to assess than using microscope. $[9,11,12,13]$ The microscopic vision often is restricted by narrow ear canals especially with a pronounced isthmus, which compels surgeons to create an angled port via post-aural approach. Here, all cases could be performed transcanal, avoiding unnecessary post-aural incision and suture clearly favouring better cosmesis and less morbidity.[12,14] Documentation and demonstration of the procedure was simpler which immensely benefits teaching. In endoscopic procedures, all the structures are seen in the same field and hence much easier to appreciate the anatomical relationship between them. Convenience and portability is an added advantage of the endoscope which is less space occupying as compared to the microscope; definite advantage for surgeons on the move.

The main disadvantage noted was that one hand had to be totally held up to stabilise the endoscope.[10] This becomes extremely cumbersome towards the later stages of surgery, especially during placement of the graft. Spreading of graft at times got messy since there was no option of anchoring the graft on one side before trying to tuck in the rest resulting in the graft moving around. Use of suction and instruments together is virtually impossible during endoscopic surgery which further complicates management of bleeding. The difficulty in graft placement got accentuated when using a wet graft (After rehydration) which was the preferred method of the author in microscopic tympanoplasty. As mentioned earlier it moved around and sometimes promptly curled up to add to the frustration. Obviously, a dry graft came to be accepted as the choice in later endoscopic surgeries.

Size of the endoscope was sometimes a limiting factor especially in narrower ear canals. The larger diameter nasal endoscope did have the advantage of wider angle of vision, but was at the cost of space. Manipulation of instruments past the endoscope got cramped up, often causing inadvertent injury to the canal wall and resultant bleeding. The 4-mm endoscope being bulkier and longer makes one handed ear surgery more difficult in comparison to the smaller and lighter ototympanoscope. Even slightest of movement of the head of the patient would result in secondary direct trauma to the canal by the tip of the endoscope; co-operation of the patient or quality of the image would be adversely affected. $[10$ 13, 14] Premedication and infiltration anaesthesia had hence to be spot on. However, one silver lining here with the use of the larger 4-mm nasal endoscope over the smaller tympanoscope is that since 'off line of sight' visualisation is possible due to its wide viewing angle, direct injury thus to middle ear structures and ossicles were virtually nil since it is almost impossible to smoothly advance the scope even beyond the canal isthmus, let aside the tympanic ring!

Presence of bleeding was another albatross around the neck during surgery; immediately soils the endoscope blurring the vision. This was further complicated by the onehanded surgery which offers no choice of doing suction and tissue manipulation together. Thermal irritation due to heat dissipation from the endoscope light was an issue for 6 patients initially and this could result in mucosal injury to the middle ear. To prevent this, the light source was used in lower settings which provided more than good illumination with less tissue heating.[15,16\} The antifog solution doubled up as an effective cooling agent for the lens which was used continuously on the tip of the endoscope. It was also noted that manipulation of the endoscope was slightly easier on the right side as compared to left; possibly related to routine 
habit of positioning on the right side during endoscopic nasal surgeries.

\section{CONCLUSION}

The $0^{0}$ wide-angled nasal endoscope is an equally effective alternative to the microscope for performing Type 1 Tympanoplasty. It provides unmatched middle ear visualisation and image quality with significant benefits in teaching and demonstration with operating time and graft uptake comparable to that of microscopic surgery. However, its use may be limited in narrow ear canals. Meticulous sterilisation of the endoscope, adequate depth of anaesthesia, use of light source in lower settings and selection of dry grafts are recommended precautions during the procedure.

\section{Acknowledgement}

I acknowledge the Audiology Department of Medical College, Kottayam for the meticulous audiometric analysis of patients pre- \& post-op.

\section{REFERENCES}

[1] Mer SB, Derbyshire AJ, Brushenko A, et al. Fiberoptic endoscopes for examining the middle ear. Arch Otolaryngol 1967;85(4):387-93.

[2] Kimura H, Yamaguchi H, Cheng SS, et al. Direct observation of the tympanic cavity by the superfine fiberscope. Nippon Jibiinkoka Gakkai Kaiho 1989;92(2):233-8.

[3] Thomassin JM, Duchon-Doris JM, Emram B, et al. Endoscopic ear surgery. Initial evaluation. Ann Otolaryngol Chir Cervicofac 1990;107:564-570.

[4] Rosenberg SI, Silverstein H, Willcox TO, et al. Endoscopy in otology and neurotology. Am J Otol 1994;15(2):168-172.

[5] Orlandi RR, Shelton C. Endoscopic closure of the eustachian tube. Am J Rhinol 2004;18(6):363-5.

[6] Marchioni D, Alicandri-Ciufelli M, Molteni G, et al. Ossicular chain preservation after exclusive endoscopic transcanal tympanoplasty: preliminary experience. Otol Neurotol 2011;32(4):626-31.
[7] McKennan KX. Endoscopic second look mastoidoscopy to rule out residual epitympanic/mastoid cholesteatoma. Laryngoscope 1993;103 (7):810-4.

[8] Marchioni D, Alicandri-Ciufelli M, Molteni G, et al. Endoscopic tympanoplasty in patients with attic retraction pockets. Laryngoscope 2010;120(9):184755.

[9] Tarabichi M. Endoscopic management of cholesteatoma: long-term results. Otolaryngol Head Neck Surg 2000;122(6):874-81.

[10] Dundar R, Kulduk E, Soy FK, et al. Endoscopic versus microscopic approach to type 1 tympanoplasty in children. Int J Pediatr Otorhinolaryngol 2014;78(7):1084-9.

[11] Ayache S, Tramier B, Strunski V. Otoendoscopy in cholesteatoma surgery of the middle ear: what benefits can be expected? Otol Neurotol 2008;29(8):1085-90.

[12] Presutti L, Marchioni D, Mattioli F, et al. Endoscopic management of acquired cholesteatoma: our experience. J Otolaryngol Head Neck Surg 2008;37(4):481-7.

[13] Kozin ED, Gulati S, Kaplan AB, et al. Systematic review of outcomes following observational and operative endoscopic middle ear surgery. Laryngoscope 2015;125(5):1205-14.

[14] Marchioni D, Villari D, Alicandri-Ciufelli M, et al. Endoscopic open technique in patients with middle ear cholesteatoma. Eur Arch Otorhinolaryngol 2011;268(11):1557-63.

[15] Bottrill I, Perrault DF Jr, Poe D. In vitro and in vivo determination of the thermal effect of middle ear endoscopy. Laryngoscope 1996;106(2 Pt 1):213-6.

[16] Kozin ED, Lehmann A, Carter M, et al. Thermal effects of endoscopy in a human temporal bone model: implications for endoscopic ear surgery. Laryngoscope 2014;124(8):E332-9. 